\title{
Evidence from Functional Neuroimaging of a Compensatory Prefrontal Network in Alzheimer's Disease
}

\author{
Cheryl L. Grady, ${ }^{1,2,3}$ Anthony R. McIntosh, ${ }^{1,2}$ Sania Beig, ${ }^{1}$ Michelle L. Keightley, ${ }^{1}$ Hana Burian, ${ }^{1}$ and Sandra E. Black ${ }^{1,4}$ \\ ${ }^{1}$ Rotman Research Institute, Baycrest Center for Geriatric Care, Toronto, Ontario, Canada M6A 2E1, and Departments of ${ }^{2}$ Psychology and ${ }^{3}$ Psychiatry, and \\ ${ }^{4}$ Cognitive Neurology Unit and Research Program in Aging, Sunnybrook and Women's College Health Sciences Centre, and Department of Medicine \\ (Neurology), University of Toronto, Toronto, Ontario, Canada M5S 1A8
}

\begin{abstract}
Previous experiments have found that individuals with Alzheimer's disease (AD) show increased activity in prefrontal regions compared with healthy age-matched controls during cognitive tasks. This has been interpreted as compensatory reallocation of cognitive resources, but direct evidence for a facilitating effect on performance has been lacking. To address this we measured neural activity during semantic and episodic memory tasks in mildly demented AD patients and healthy elderly controls. Controls recruited a left hemisphere network of regions, including prefrontal and temporal cortices in both the semantic and episodic tasks. Patients engaged a unique network involving bilateral dorsolateral prefrontal and posterior cortices. Critically, activity in this network of regions was correlated with better performance on both the semantic and episodic tasks in the patients. This provides the most direct evidence to date that AD patients can use additional neural resources in prefrontal cortex, presumably those mediating executive functions, to compensate for losses attributable to the degenerative process of the disease.
\end{abstract}

Key words: semantic memory; episodic memory; dementia; vision; neuroimaging; positron emission tomography; frontal lobe

\section{Introduction}

Early in the course of Alzheimer's disease (AD), deficits are found in both semantic and episodic memory (Weingartner et al., 1993; Perry and Hodges, 2000). There is evidence that some aspects of the structure of semantic memory may be relatively intact (Ober and Shenaut, 1999), but access to specific information about object attributes is lost (Hodges et al., 1992; Binetti et al., 1995; Giffard et al., 2001). Episodic memory is affected most dramatically for recently acquired information (Welsh et al., 1991), but retrieval of remote memories also may be impaired (Greene and Hodges, 1996). Functional neuroimaging studies have shown that activity in specific brain areas known to participate in semantic and episodic memory in healthy individuals (Cabeza and Nyberg, 2000) is related to memory ability in AD patients. For example, semantic processing in $\mathrm{AD}$ patients is correlated with activity in left hemisphere lateral temporal, parietal, and prefrontal regions (Grossman et al., 1997; Desgranges et al., 1998) and also with activity in left anterior prefrontal cortex (Saykin et al., 1999). Episodic memory in AD patients is correlated with increased activity in temporoparietal regions (Grady et al., 1988; Desgranges et al., 1998; Stern et al., 2000) and in medial temporal regions (Desgranges et al., 1998; Eustache et al., 2001). In some cases these posterior regions are correlated with memory performance only in the patients and not in healthy controls (Stern et

\footnotetext{
Received Aug. 22, 2002; revised Nov. 4, 2002; accepted Nov. 18, 2002.

This work was funded by the Canadian Institutes of Health Research (operating Grants 14036 and 13129) and the Alzheimer Society of Canada. We thank the staff at the Positron Emission Tomography Centre of the Centre for Addiction and Mental Health, University of Toronto, for their assistance in this experiment. We also thank Maureen Evans for her help in patient recruitment.

Correspondence should be addressed to Cheryl L. Grady, Rotman Research Institute, Baycrest Centre for Geriatric Care, 3560 Bathurst Street, Toronto, Ontario, Canada M6A 2E1. E-mail: cgrady@rotman-baycrest.on.ca.

Copyright $\odot 2003$ Society for Neuroscience $\quad 0270-6474 / 03 / 230986-08 \$ 15.00 / 0$
}

al., 2000). These correlations also are dependent in part on dementia severity, because correlations between verbal episodic memory and activity in medial temporal regions have been found in mildly demented patients, and between memory scores and left temporal cortex activity in more severely impaired patients (Desgranges et al., 2002).

One of the more interesting findings from neuroimaging studies of early AD is that of increased prefrontal activity during some cognitive tasks compared with older controls (Becker et al., 1996; Woodard et al., 1998; Backman et al., 1999; Saykin et al., 1999). Increased functional connectivity of prefrontal regions, as defined by the correlations among measures of activity in these areas (Friston et al., 1993; Horwitz, 1994; McIntosh, 1999), also has been reported in $\mathrm{AD}$ patients compared with controls (Horwitz et al., 1995). Although these increases in prefrontal activity and functional connectivity have been interpreted as compensatory reallocation or recruitment of cognitive resources, a direct link between altered prefrontal activity and ability to perform these tasks has been lacking. It would be particularly important to be able to relate prefrontal function to preserved memory ability in $\mathrm{AD}$ patients, because these areas are typically affected later in the course of the disease (Grady et al., 1988; Jagust et al., 1988), and they mediate organizational and executive functions (Stuss and Benson, 1984; D'Esposito et al., 1995; Fuster, 2000) that might operate across multiple types of task. The purpose of the current experiment was to examine the neural correlates of semantic and episodic memory in patients with $\mathrm{AD}$, including the assessment of functional connectivity to identify "cognitive networks," and to relate activity in these networks to performance. Specifically, we looked for evidence that those patients who were able to recruit prefrontal cortex to a greater degree during these tasks would perform more accurately, which would directly support the idea that activity in these regions is compensatory. 


\section{Materials and Methods}

Twelve older healthy adults ( 8 men, 4 women; 10 right-handed, 2 lefthanded) and 12 mildly demented patients with probable AD (10 men, 2 women; all but 1 right-handed) participated in the experiment. All of the healthy controls were screened to rule out any diseases or medications that might affect brain function. All participants underwent structural magnetic resonance imaging scans to rule out strokes or other abnormalities aside from generalized atrophy. All patients were taking medication for their cognitive impairments (seven on Aricept, two on Exelon, one on Galantamine, and one on Propentofylline). Four of the male patients had a history of cardiovascular problems, but otherwise there were no patients with diseases that would compromise brain function aside from the dementia. The two groups showed no significant differences in age or years of education (see Table 1). Subjects needing correctional lenses to view the stimuli wore their own glasses during the experiment. This experiment was approved by the ethics committees of Baycrest Centre for Geriatric Care and Sunnybrook and Women's College Health Science Centre and conducted with the written consent of each participant. One male AD patient was an outlier on the task analysis of brain activity described below and was removed from all analyses, resulting in 9 males and 2 females in this group. Scores for the AD patients on the neuropsychological tests (see Table 1) were compared with a separate control group that was not a part of the imaging study $(n=50$; age $=70.5 \pm 6.6$ years; education $=14.8 \pm 2.9$ years).

Stimuli and tasks. Stimuli for this experiment consisted of black line drawings of common objects and words representing the names of objects (Snodgrass and Vanderwart, 1980) presented on a white background. Each task consisted of 15 trials, and practice trials preceded each task. During each trial in the semantic tasks, a word or object appeared on either the right or left side of the computer screen, and a visual noise pattern appeared on the other side. Participants were instructed to make a living/nonliving decision about each object or word and press the left mouse button if the object/word represented something living and the right button if it were nonliving. The baseline tasks involved presentation of novel objects or words, and participants were instructed to press the button corresponding to the side of the screen on which the word/object appeared. During the recognition tasks, objects or words were presented in both positions on the screen (one new stimulus and one that was seen previously during the semantic tasks), and participants pressed the button corresponding to the side of the screen on which the "old" item was presented. During all conditions, each trial lasted $4 \mathrm{sec}$ with a $1 \mathrm{sec}$ intertrial interval (blank screen). Half of the participants received the object tasks before the word tasks, and half received the word tasks first. All tasks were presented via a PC running SuperLab on a computer monitor suspended above the scanner bed (Cedrus, Phoenix, AZ).

Scanning procedure. Six positron emission tomography (PET) scans, with injections of $40 \mathrm{mCi}$ of $\mathrm{H}_{2}{ }^{15} \mathrm{O}$ each and separated by $11 \mathrm{~min}$, were performed on all participants. Scans 1 and 6 consisted of presentations of the control tasks, scans 2 and 3 were semantic tasks, and scans 4 and 5 were recognition tasks. Scans were performed on a General Electric Medical Systems PC2048-15B tomograph, which has a reconstructed resolution of $6.5 \mathrm{~mm}$ in both transverse and axial planes. This tomograph allows 15 planes, separated by $6.5 \mathrm{~mm}$ (center to center), to be acquired simultaneously. Emission data were corrected for attenuation by means of a transmission scan obtained at the same levels as the emission scans. Head movement during the scans was minimized with a thermoplastic mask that was molded to each person's head and attached to the scanner bed. Before each scan the instructions for the task to be performed during that scan were read to the participant. Estimates of regional cerebral blood flow ( $\mathrm{rCBF}$ ) were obtained from the measured radioactivity counts in each scan (Herscovitch et al., 1983).

Image analysis. Each participant's PET scans were registered to the first scan to correct for small movements during the scanning session using automated image registration (Woods et al., 1992). Images were then spatially normalized to the Talairach and Tournoux (1988) atlas coordinate system and smoothed using a $10 \mathrm{~mm}$ filter (to increase signal to noise and reduce the effects of individual differences in anatomy) using SPM95 (Frackowiak and Friston, 1994). Ratios of rCBF to global CBF within each scan for each subject were computed, and the effects of any global rCBF differences between the groups were removed by regressing out the group main effect from each voxel for each subject, leaving only the residual variance that was caused by the tasks.

There were three steps in the image analysis. The first was to test for modulations of brain activity attributable to the task conditions and identify a brain area that could serve as the reference region for further functional connectivity analyses. The choice of region was based on three criteria: (1) location in prefrontal cortex, (2) reliable modulation of activity across tasks in one or both groups, and (3) previous evidence for a role of this region in the cognitive processes under study. The second step was to identify the cognitive networks that were active during semantic and episodic memory tasks by determining the areas of the brain in which activity was correlated with that of the reference region, i.e., its functional connectivity (Friston et al., 1993; Horwitz, 1994; McIntosh, 1999). The final step was to determine whether activity in these networks was correlated with behavioral performance on the tasks. In this analysis we tested the hypothesis that the network of regions identified in the preceding functional connectivity analysis would be correlated, as a whole, with behavior.

Because all of these analytical steps are based on the assumption that cognition is the result of the integrated activity of dynamic brain networks rather than the action of any single region acting independently, our approach to image data analysis was designed to reveal these networks through multivariate techniques. To this end, all analyses were performed using partial least squares (PLS) (McIntosh et al., 1996), which is a multivariate analysis that identifies groups of brain regions distributed over the entire brain that together covary with some aspect of the experimental design. This is in contrast to the more typically used univariate analysis that assesses the significance of each region separately. PLS operates on the covariance between brain voxels and the experimental design, or a set of external measures, to identify a new set of variables [so-called latent variables (LVs)]. The results of PLS analysis are expressed in terms of LVs, each of which identifies a pattern of differences in brain activity across the tasks and the brain voxels showing this effect. Each brain voxel has a weight on each LV, known as a salience, that indicates how that voxel is related to the LV. A salience can be positive or negative, depending on whether the voxel shows a positive or negative relation with the pattern identified by the LV. Multiplying the rCBF value in each brain voxel for each subject by the salience for that voxel, and summing across all voxels, gives a latent variable score (called a "brain" score here) for each subject for each task condition on a given LV. The brain scores can be used to examine differences in brain activity across conditions, because greater activity in brain areas with positive (or negative) weights on a latent variable will yield positive (or negative) mean scores for a given condition. In addition, these scores can be correlated with external variables (see below).

The significance of each LV was assessed using a permutation test (Edgington, 1980; McIntosh et al., 1996), using $p<0.01$ as the statistical threshold. In addition to the permutation test, we determined the reliability of the saliences for the brain voxels characterizing each LV. To do this, all saliences in each analysis were submitted to a bootstrap estimation of the SEs (Efron and Tibshirani, 1986; Sampson et al., 1989). A reliable contribution for a given voxel was defined as a ratio of salience to $\mathrm{SE} \geq 3$, which corresponds to the $99 \%$ confidence interval (Sampson et al., 1989). Because all saliences are calculated in a single analytical step, there is no need to correct for multiple comparisons, as is often done in univariate analyses (McIntosh et al., 1996). Local maxima for the reliable brain areas on each LV were defined as the voxel with a ratio higher than any other voxel in a $2 \mathrm{~cm}$ cube centered on that voxel. Locations of these maxima are reported in terms of brain region, or gyrus, and estimated Brodmann area (BA) as defined in the Talairach and Tournoux atlas (1988).

For the task analysis, data from both patients and controls were entered (i.e., there was one task analysis that assessed differences across all six tasks in both groups simultaneously). The reference region, or "seed," chosen for the second step of examining functional connectivity was identified from the results of the task PLS analysis. Because our hypothesis was that activity in prefrontal cortex would be critical to task perfor- 
mance, we chose a region of left ventrolateral prefrontal cortex (VLPFC) that showed highly reliable task-related changes in both patients and controls. However, because an additional region in left dorsal occipital cortex showed the same pattern of task-related activity, both areas were included in the connectivity analysis (see Results for the regions used as the seeds). For the functional connectivity analysis, data from the semantic and recognition tasks in both groups were used (the baseline tasks were not used in this analysis). Connectivity was determined by means of a "seed voxel" analysis (Schreurs et al., 1997; McIntosh, 1999) in which the rCBF values from the seeds are extracted, and the correlations between seed activity and activity in all brain voxels within each condition were calculated. PLS was used to contrast these correlations across conditions and between patients and controls. Thus, with this analysis it can be seen whether the brain regions whose activity is correlated with activity in the seed are the same or different between groups and across task conditions. Permutation and bootstrap analyses were performed on the resulting LVs as described above. In addition, the correlations between the brain scores from each significant LV and the seed rCBF values were calculated to assess the relation between the whole brain pattern and activity in the two reference regions. The reliability of each of these correlations in each condition was assessed by calculating confidence intervals via the bootstrap.

The final analytical step was to use PLS to assess the correlations between activity in the network identified in the connectivity analysis and accuracy of task performance in the patients (Grady et al., 2001a). The correlations between task accuracy and brain activity in controls could not be examined because the majority of controls performed at or near ceiling on all tasks. This analysis involved assessing simultaneously the brain areas in which activity was correlated with activity in both seed regions and with the behavioral measure, i.e., a combined network connectivity and behavior analysis. To confirm that the resulting patterns of correlations from this combined analysis characterized both the seeds and performance, a separate analysis of performance by itself also was conducted for comparison.

\section{Results}

The $\mathrm{AD}$ patients were significantly impaired on standard neuropsychological tests of episodic memory, executive function, and

Table 1. Demographic and neuropsychological data

\begin{tabular}{|c|c|c|}
\hline Measure & Controls & AD patients \\
\hline \multicolumn{3}{|l|}{ Demographics $^{a}$} \\
\hline Age (years) & $71 \pm 4$ & $74 \pm 9$ \\
\hline Education (years) & $15 \pm 4$ & $15 \pm 3$ \\
\hline Mini mental status exam & $28 \pm 1$ & $22 \pm 5^{*}$ \\
\hline Mattis dementia rating scale & & $117 \pm 11$ \\
\hline Duration of illness (years) & & $5 \pm 3$ \\
\hline \multicolumn{3}{|l|}{ Memory $^{b}$} \\
\hline Mattis DRS memory scale & $24 \pm 1$ & $15 \pm 3^{*}$ \\
\hline CVLT acquisitionc & $50 \pm 10$ & $21 \pm 10^{*}$ \\
\hline Digit span forward & $9 \pm 2$ & $8 \pm 2$ \\
\hline Digit span backward & $7 \pm 2$ & $5 \pm 2$ \\
\hline \multicolumn{3}{|l|}{ Language/Semantic $^{b}$} \\
\hline Boston naming test & $28 \pm 2$ & $19 \pm 7^{*}$ \\
\hline FAS fluency & $47 \pm 15$ & $29 \pm 10^{*}$ \\
\hline Semantic fluency & $20 \pm 5$ & $11 \pm 4^{*}$ \\
\hline \multicolumn{3}{|l|}{ Visuospatial $^{b}$} \\
\hline Rey-0sterrieth figure (copy) & $33 \pm 3$ & $28 \pm 10$ \\
\hline Line orientation & $25.9 \pm 3.7$ & $25.6 \pm 2.2$ \\
\hline \multicolumn{3}{|l|}{ Executive function ${ }^{b}$} \\
\hline WCST (number of categories) & $3.8 \pm 0.8$ & $1.4 \pm 2.1^{*}$ \\
\hline Raven's matrices & $32 \pm 3$ & $28 \pm 5$ \\
\hline Trailmaking test $\mathrm{B}$ (sec) & $72 \pm 34$ & $184 \pm 53^{*}$ \\
\hline
\end{tabular}

Values are mean \pm SD. ${ }^{*}$ Significantly different from controls by $t$ test; $p<0.01$. CVLT, California verbal learning test: WCST, Wisconsin card sorting test.

${ }^{a}$ Data for subjects in the imaging study.

${ }^{b}$ Separate control group (see Materials and Methods).

'Standard $t$ score. semantic processing (Table 1). The patients were not impaired as a group on tests of working memory (i.e., digit span) and visuospatial function. Performance on the semantic and recognition tasks performed during scanning is shown in Figure 1. Patients performed significantly less accurately on these tasks than did the controls (repeated measures ANOVA; $F=78.4 ; p<0.001$ ), as expected. However, the range of scores was quite large in the patients, with some performing poorly and others performing within the normal range on the semantic tasks. Response times were not significantly different between groups $(F=4.0 ; p=$ 0.06) (Fig. 1), although there was a trend for the patients to respond more slowly.

The first two LVs from the task analysis (Fig. 2) show patterns of activity that are common to both patients and controls. The first LV $(p<0.001)$ distinguished recognition of words and objects from the semantic and baseline tasks (Fig. 2B). Recognition was characterized by increased activity bilaterally in prefrontal regions (although more extensive in the left hemisphere), visual and parietal cortices, and medial temporal regions (Fig. 2A; Table 2). Decreased activity during recognition, relative to the other tasks, was found in perisylvian regions and medial prefrontal cortex. The second LV $(p=0.002)$ differentiated both the semantic retrieval and recognition tasks from baseline, particularly when words were presented (Fig. 2D). During both the semantic and recognition tasks, there was increased activity in left prefrontal, temporal, and dorsal extrastriate regions, compared with the baseline task (Fig. 2C; Table 2), and decreased activity in
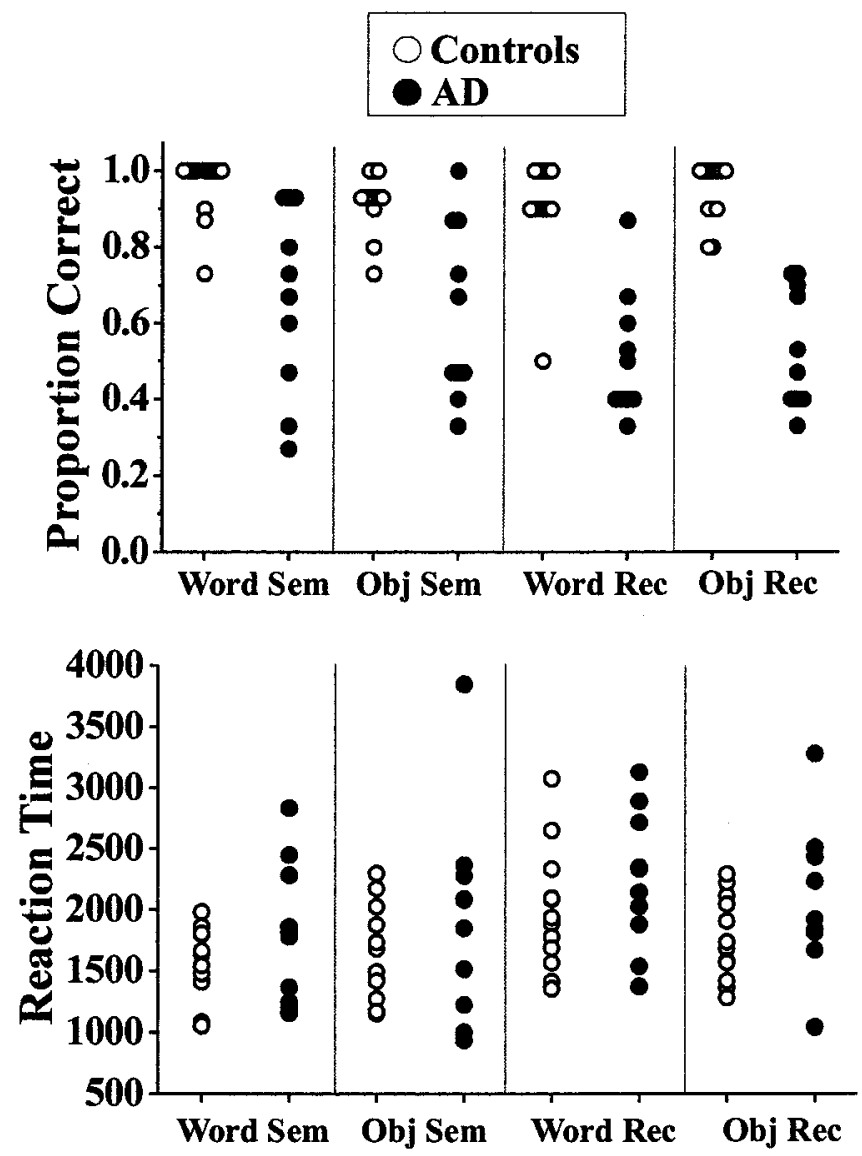

Figure 1. Scatter plots of performance on the semantic and recognition tasks. Task accuracy below chance performance (50\%) in some patients was caused by failures to respond to some items. Sem, Semantic task; Rec, recognition task; Obj, object. 


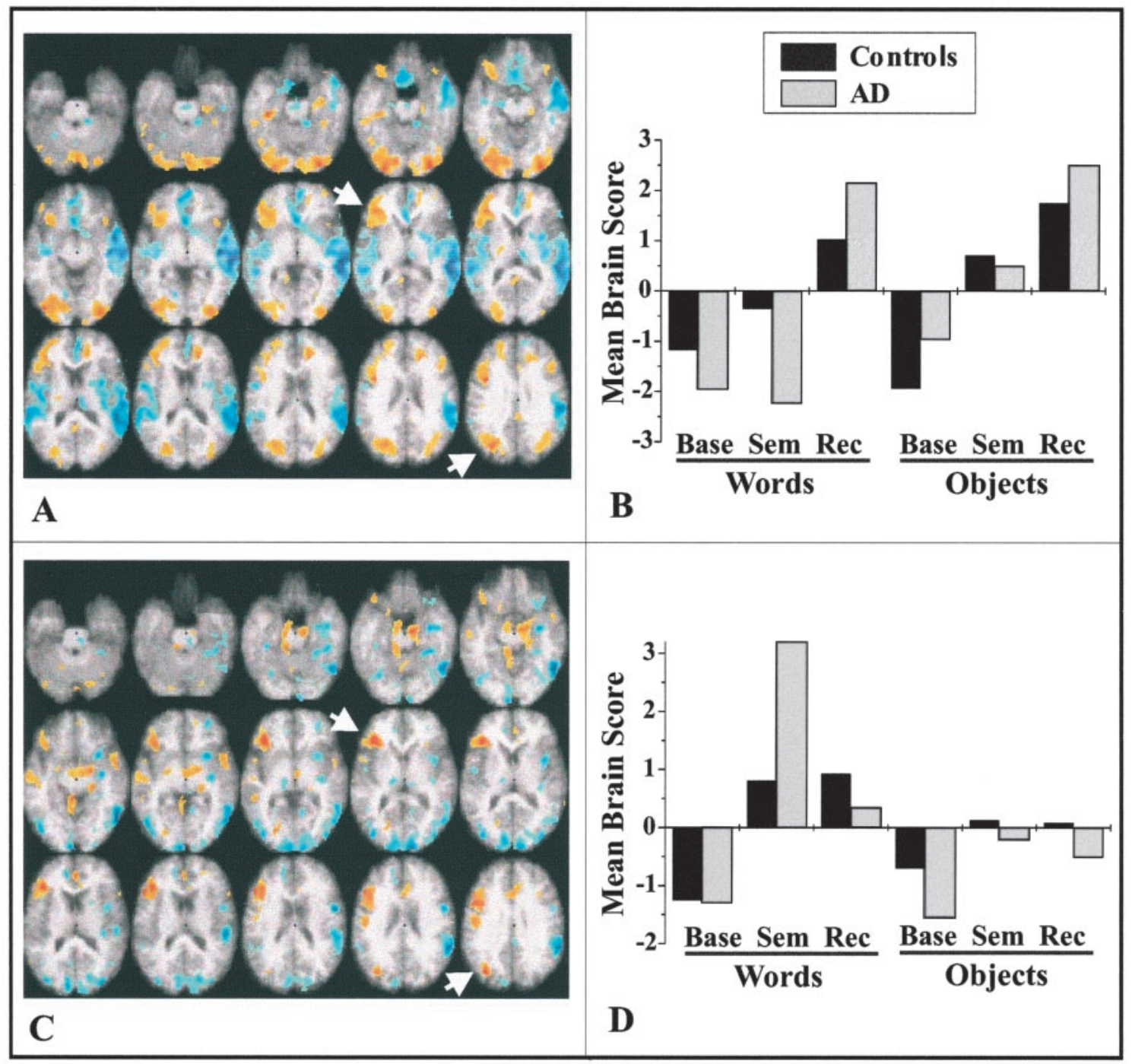

Figure 2. Changes in brain activity related to task for controls and AD patients. The images in $A(L V 1 ; p<0.001)$ and $C(L V 2 ; p=0.002)$ show the active areas on a standard magnetic resonance imaging scan in which the right side of the brain is shown on the right side of the image. The brain slices begin at $-28 \mathrm{~mm}$ relative to the anterior commissure-posterior commissure line (top left image) and end at $+28 \mathrm{~mm}$ (bottom right image) with a $4 \mathrm{~mm}$ slice separation. The graphs in $B$ and $D$ show the mean brain scores for controls and AD patients on the LVs. Positive mean brain scores were found in those conditions in which activity was increased in the brain regions shown in red and yellow (i.e., those with positive salience on the LV). Negative mean brain scores were found in those conditions in which activity was increased in the brain regions shown in blue (those with negative salience on the LV). Arrows point to the regions of left VLPFC and extrastriate cortex used in subsequent analyses. Maxima of regions with increased activity during the semantic and recognition tasks (salience/SE $\geq 3.0$ ) are shown in Table 2. Base, Baseline task; Sem, semantic task; Rec, recognition task.

ventral and medial extrastriate visual areas and in some right temporal and parietal regions.

The most prominent prefrontal region with changes in activity related to the task conditions was a region of VLPFC that showed a highly reliable contribution to both LVs (Fig. $2 \mathrm{~A}, \mathrm{C}$, arrows; Table 2). Thus, although the overall patterns of activity were different across LVs, this prefrontal region contributed to episodic recognition as well as retrieval from semantic memory in both patients and controls. Interestingly, an additional region in left dorsal extrastriate cortex [superior occipital gyrus (GOs)] showed the same pattern of activity as left VLPFC (Fig. 2A, C, arrows; Table 2). Therefore, to identify the cognitive networks that are active during semantic and episodic memory, we examined the joint functional connectivity of these two areas. The first LV from this connectivity analysis identified a pattern of correlations that characterized the controls but not the patients $(p<$ 0.001 ) (Fig. 3A). The correlations between activity in each of the seeds and the brain score, which is a weighted average of activity in all brain voxels, were positive in the control group across all task conditions (Table 3). None of these correlations was reliable in the patients. In controls, activity in left VLPFC and GOs was correlated primarily with activity in other left hemisphere regions, similar to those that differentiated both semantic and episodic tasks from baseline (compare Figs. $2 C, 3 A$ ). That is, a network of positively intercorrelated left hemisphere regions was identified that included VLPFC, dorsal occipital cortex, middle temporal gyrus $(x,-56 ; y,-50 ; z, 0$; reliability ratio $=5.4)$, left lingual gyrus $(x,-6 ; y,-64 ; z,-8$; ratio $=5.2)$, and the left insula $(x,-32 ; y,-6 ; z, 16 ;$ ratio $=4.1)$. Negative correlations were found in a few regions, including the right middle temporal gyrus and the anterior cingulate.

The second LV from the functional connectivity analysis identified a pattern characterizing the patients but not the controls $(p<0.001)$ (Fig. 3B). The correlations between VLPFC and GOs 
Table 2. Brain areas with task-related increases of activity in both AD patients and controls

\begin{tabular}{|c|c|c|c|c|c|c|}
\hline Region, gyrus & Hem & BA & $x$ & $y$ & $z$ & Ratio \\
\hline \multicolumn{7}{|c|}{ Increases during recognition task (LV1) } \\
\hline \multicolumn{7}{|l|}{ Prefrontal } \\
\hline GFi & L & $45 / 47$ & -38 & 32 & 4 & 6.1 \\
\hline GFm & $\mathrm{R}$ & 9 & 40 & 20 & 28 & 4.0 \\
\hline GFm & L & 9 & -38 & 12 & 36 & 4.5 \\
\hline Cingulate & $\mathrm{R}$ & 32 & 8 & 12 & 40 & 6.4 \\
\hline Hippocampus & $\mathrm{R}$ & & 28 & -16 & -20 & 4.0 \\
\hline Parahippocampal gyrus & L & 36 & -36 & -30 & -20 & 6.8 \\
\hline \multicolumn{7}{|l|}{ Extrastriate } \\
\hline GL & $\mathrm{R}$ & 18 & 26 & -88 & -16 & 7.8 \\
\hline GF & L & 18 & -28 & -92 & -16 & 7.2 \\
\hline GOm & $\mathrm{R}$ & 18 & 26 & -84 & 20 & 4.5 \\
\hline GOs & L & 19 & -30 & -72 & 28 & 6.8 \\
\hline Parietal (LPi) & L & 40 & -40 & -58 & 40 & 4.7 \\
\hline \multicolumn{7}{|c|}{ Increases during semantic and recognition tasks (LV2) } \\
\hline \multicolumn{7}{|l|}{ Prefrontal } \\
\hline GFi & L & 47 & -36 & 28 & 4 & 5.5 \\
\hline GFi & L & 44 & -46 & 8 & 28 & 4.5 \\
\hline Temporal (GTs) & $\mathrm{R}$ & 38 & 48 & 0 & -8 & 3.5 \\
\hline Parahippocampal gyrus & $\mathrm{R}$ & 28 & 20 & -28 & -8 & 3.8 \\
\hline Cingulate & M & 24 & -2 & 20 & 28 & 3.6 \\
\hline Extrastriate (GOs) & L & 19 & -34 & -72 & 28 & 5.0 \\
\hline
\end{tabular}

$x$ (right/left), Negative values are in the left hemisphere; $y$ (anterior/posterior), negative values are posterior to the zero point (located at the anterior commissure); $z$ (superior/inferior), negative values are inferior to the plane defined by the anterior and posterior commissures. Hem, Hemisphere; $\mathrm{R}$, right; L, left; $\mathrm{M}$, midline ( $\leq 5 \mathrm{~mm}$ from 0 point in $x$ dimension); $\mathrm{BA}$, Brodmann's area; Ratio, salience/SE ratio from the bootstrap analysis; $\mathrm{GF}$, fusiform gyrus; $G F(\mathrm{~m}, \mathrm{i})$, frontal gyrus (middle, inferior); $\mathrm{GL}$, lingual gyrus; $\mathrm{GO}(\mathrm{m}, \mathrm{s})$, occipital gyrus (middle, superior); $\mathrm{GTs}$, superior temporal gyrus; LPi, inferior parietal.

activity and the brain scores in the patients were positive and reliable across all four tasks (Table 3) but not reliable in the controls. Similar to the controls, the patients showed positive correlations among left hemisphere frontal, occipital, and temporal areas. In addition, the patients showed extensive regions of right frontal and temporoparietal areas where activity was correlated with activity in both seeds, areas that were not reliably correlated in controls. This indicates a wider recruitment of prefrontal cortex into this cognitive network in the patients, similar to that previously reported, but does not in itself provide a link between this recruitment and task performance. To determine the existence of such a link, we tested the hypothesis that activity in this network of regions would be associated with better memory performance by including activity from the two seed regions and accuracy in the same correlational analysis. This resulted in a single significant LV $(p<0.001)$ that identified regions where there were positive correlations with all three variables, i.e., with both seeds and performance in the semantic and episodic tasks. Positive correlations with all three variables were seen in bilateral dorsolateral prefrontal cortex, anterior cingulate gyrus, and right temporoparietal cortex (Table 4). To confirm that each of these regions from the combined analysis was correlated separately with brain activity and performance, the bootstrap ratios for each area were extracted from these separate analyses and are shown in Table 4.

\section{Discussion}

In this experiment we identified regions with increases in activity that were specific to episodic recognition, such as the medial temporal regions, as well as regions that were active during both semantic and recognition tasks, such as left VLPFC and dorsal extrastriate cortex. The changes in brain activity related to task that we observed in our patient and control groups were consistent with those found in earlier experiments. For example, dorsolateral prefrontal, parietal, and medial temporal regions commonly show increased activity during episodic retrieval tasks (for review, see Cabeza and Nyberg, 2000). Similarly, the left VLPFC and temporal areas that were active in the semantic task used here are consistently activated in other lexical and semantic tasks (Vandenberghe et al., 1996; Duzel et al., 1999; Wiggs et al., 1999; Braun et al., 2001; Wagner et al., 2001). Although some have found more right prefrontal activity during episodic retrieval and left prefrontal activity during semantic retrieval or encoding of new material (Tulving et al., 1994), we found more left prefrontal activity in both kinds of tasks. This is likely because of the fact that some encoding of new items was necessary during the recognition task (Buckner et al., 2001).

There are two novel contributions from this experiment. The first is that the brain networks underlying semantic and episodic memory, as characterized by the functional connectivity of left hemisphere frontal and occipital areas, were altered in mildly demented $\mathrm{AD}$ patients compared with healthy older controls. In controls, a network including left VLPFC, dorsal extrastriate cortex, and temporal areas was identified, whereas in patients a more extensive network of regions was recruited, including bilateral prefrontal and temporoparietal cortices. Critically, activity in this network of regions was correlated with the ability of the patients to perform the tasks accurately. That is, those patients who had more activity in bilateral prefrontal areas were better able to perform tasks of semantic and episodic memory. This is thus the first direct demonstration that recruitment of additional prefrontal areas into a cognitive network in $\mathrm{AD}$ patients is associated with better performance. However, this facilitating effect is not limited to prefrontal cortex but includes temporal and parietal areas similar to those found previously to be correlated with semantic and episodic memory performance in AD patients (Grossman et al., 1997; Desgranges et al., 1998) and thought to mediate memory storage and retrieval (Nyberg et al., 1995; Wilding and Rugg, 1996; Smith and Jonides, 1998). This suggests that it is activity in the network as a whole that is compensatory and that this activity may serve to facilitate or maintain interactions among posterior 


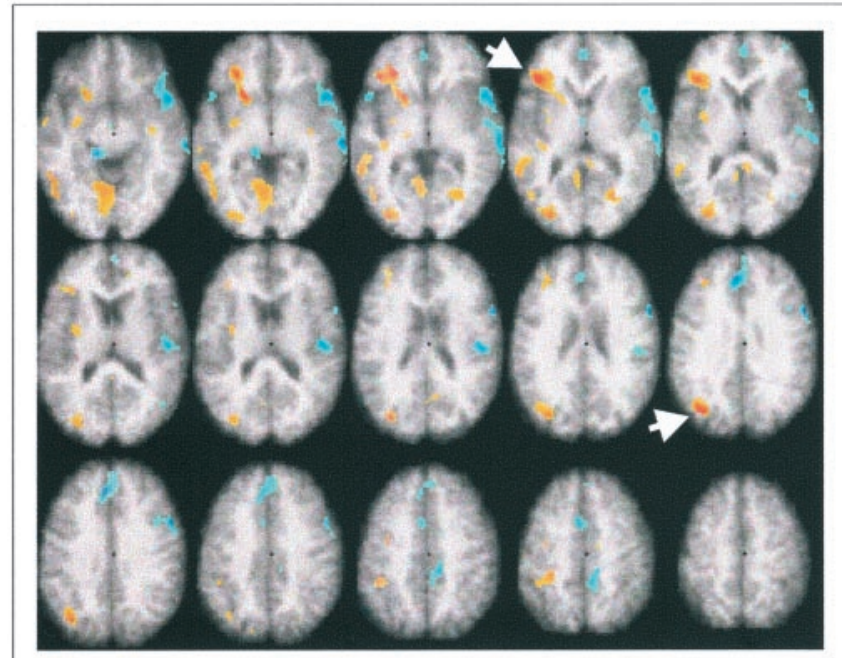

\section{A. Controls}

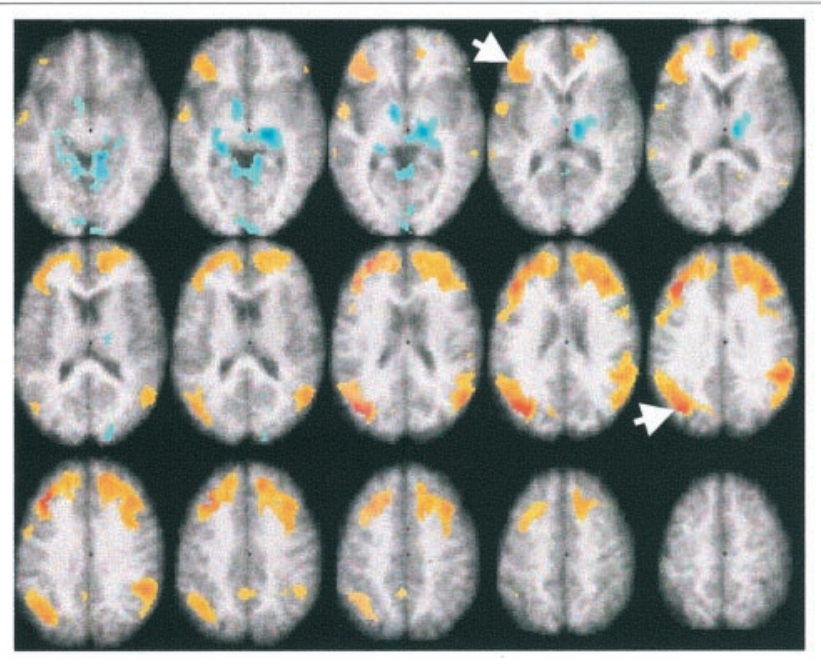

\section{B. AD patients}

Figure 3. Functional connectivity of left VLPFC and GOS. A, Connectivity in the control group; $B$, connectivity in the AD patients. The VLPFC voxel in both groups was $x-36, y 28$, and $z 4$, and for the extrastriate region the voxel used was $x-34, Y-72$, and $z 28$ (indicated by white arrows). Positive correlations are shown in yellow and red, and negative correlations are shown in blue. Maxima of regions with positive correlations for the controls (salience/SE $\geq 3.0$ ) are given in Results, and maximum regions of positive correlation for the AD patients are shown in Table 4.

Table 3. Results of the functional connectivity analysis

\begin{tabular}{lllll}
\hline Region & Word sem & Word rec & Object sem & 0bject rec \\
\hline $\begin{array}{l}\text { Controls } \\
\text { Left VLPFC }\end{array}$ & $0.61^{\dagger}$ & $0.63^{\dagger}$ & $0.65^{\dagger}$ & $0.67^{\dagger}$ \\
$\quad$ Left G0s & $0.71^{\dagger}$ & $0.70^{*}$ & $0.92^{\dagger}$ & $0.85^{\dagger}$ \\
$\begin{array}{l}\text { AD Patients } \\
\text { Left VLPFC }\end{array}$ & $0.59^{\dagger}$ & $0.52^{*}$ & $0.66^{\dagger}$ & $0.65^{\dagger}$ \\
Left G0s & $0.90^{\dagger}$ & $0.87^{\dagger}$ & $0.87^{\dagger}$ & $0.95^{\dagger}$
\end{tabular}

*Reliable at the $95 \%$ confidence interval; ${ }^{\dagger}$ reliable at the $99 \%$ confidence interval. Correlations are between each variable and the brain scores from the LVs that characterized controls and patients, respectively. GOs, Superior occipital gyrus; sem, semantic task; rec, recognition task.

storage regions and prefrontal areas mediating executive and monitoring functions.

The finding that the altered connectivity pattern in the patients was the same across task conditions suggests that increased
Table 4. Brain areas where activity is positively correlated with left VLPFC, left GOS, and task accuracy in AD patients

\begin{tabular}{lcrrrrrrr}
\hline Region, gyrus & Hem & \multicolumn{1}{c}{ BA } & \multicolumn{1}{c}{$x$} & $y$ & $z$ & Comb. & Seeds & Acc. \\
\hline Prefrontal & & & & & & & & \\
$\quad$ GFi & $\mathrm{L}$ & 47 & -38 & 26 & 4 & 7.4 & 6.4 & 2.7 \\
$\quad$ GFm & $\mathrm{R}$ & 46 & 38 & 28 & 28 & 9.8 & 7.1 & 4.0 \\
$\quad$ GFs & $\mathrm{L}$ & 9 & -24 & 48 & 24 & 10.0 & 6.1 & 2.6 \\
Cingulate & $\mathrm{R}$ & 32 & 8 & 44 & 4 & 8.2 & 6.1 & 4.2 \\
& $\mathrm{~L}$ & 32 & -18 & 26 & 40 & 6.2 & 7.2 & 4.2 \\
Temporal (GTm) & $\mathrm{R}$ & 39 & 46 & -60 & 16 & 8.8 & 6.9 & 4.4 \\
Extrastriate (GOS) & $\mathrm{L}$ & 19 & -36 & -76 & 24 & 11.4 & 14.0 & 3.9 \\
Parietal (Gsm) & $\mathrm{R}$ & 40 & 50 & -42 & 28 & 11.9 & 9.8 & 3.9 \\
\hline
\end{tabular}

Coordinates are from the combined analysis of left VLPFC and G0s seeds and accuracy. $x$ (right/left), Negative values are in the left hemisphere; $y$ (anterior/posterior), negative values are posterior to the zero point (located at the anterior commissure); $z$ (superior/inferior), negative values are inferior to the plane defined by the anterior and posterior commissures. Hem, Hemisphere; $\mathrm{R}$, right; $\mathrm{L}$, left; $\mathrm{M}$, midline ( $\leq 5 \mathrm{~mm}$ from 0 point in $x$ dimension); $B A$ Brodmann's area; Comb., salience/SE ratio from the combined analysis of seeds and accuracy; Seeds, salience/SE ratio from the connectivity analysis of the frontal and occipital seeds; Acc., salience/SE ratio from the accuracy analysis; GF(i,m,s), frontal gyrus (inferior, middle, superior); GOs, superior occipital gyrus; Gsm, supramarginal gyrus; GT(m), middle temporal gyrus.

Table 5. Common prefrontal areas from two experiments where increased activity is correlated with better memory performance in AD patients

\begin{tabular}{lcrrrrll}
\hline Gyrus & Hem & BA & \multicolumn{1}{c}{$x$} & $y$ & \multicolumn{1}{c}{$z$} & Current $^{a}$ & $\begin{array}{l}\text { Face working } \\
\text { memory }^{a}\end{array}$ \\
\hline GFi & $\mathrm{L}$ & 47 & -38 & 26 & 0 & 6.5 & 3.1 \\
GFm & $\mathrm{L}$ & 46 & -38 & 42 & 12 & 7.5 & 6.8 \\
GFm & $\mathrm{R}$ & 10 & 22 & 52 & 12 & 4.7 & 3.8 \\
GFd & $\mathrm{R}$ & 9 & 6 & 48 & 24 & 5.9 & 4.1
\end{tabular}

Coordinates are from the overlap of the bootstrap ratios from the current experiment and from a previously published face working memory experiment (Grady et al., 2001b), calculated by multiplying the two images. $x$ (right/ left), Negative values are in the left hemisphere; $y$ (anterior/posterior), negative values are posterior to the zero point (located at the anterior commissure); $z$ (superior/inferior), negative values are inferior to the plane defined by the anterior and posterior commissures. Hem, Hemisphere; R, right; L, left; BA, Brodmann's area; GF(i,m,d), frontal gyrus (inferior, middle, medial).

${ }^{a}$ Salience/SE ratios from the separate analyses from the two experiments.

recruitment of prefrontal regions is not task specific but could reflect a more general adaptation to loss of cognitive resources. Support for this idea also can be found by comparing the frontal areas associated with better performance on the semantic and recognition tasks with areas associated with better face working memory performance from a previous study (Grady et al., $2001 b$ ). In that earlier study, the focus was on brain regions with both task-related changes in activity and correlations with performance measures. Right ventral prefrontal cortex showed increased activity in patients and controls during the tasks, and this activity was correlated with better memory performance in both groups. The interest here was to determine whether there might be other prefrontal areas in which activity was correlated with performance, not previously identified, that would overlap with those seen in the current experiment. To determine these common areas, we calculated the overlap of the images obtained from the combined seed/accuracy analysis from the current experiment and a PLS analysis of the correlations between brain activity and task accuracy from the working memory experiment [for a description of this procedure see Grady et al. (2001a)]. Common areas in both right and left middle frontal gyri were positively correlated with task performance in both experiments, as was activity in left VLPFC (Table 5). This provides converging evidence from both verbal and nonverbal memory tasks that patients in the early stages of $\mathrm{AD}$ who are able to recruit these prefrontal regions are able to perform at a higher level. The compensatory effect of activity in these regions may thus reflect utilization of general cognitive resources rather than resources specific to a particular task. Evidence that recruitment of prefrontal 
regions is related to task effort or complexity across different kinds of tasks, such as semantic (Maril et al., 2001), working memory (Braver et al., 1997), or perceptual tasks (Grady et al., 1996), is consistent with this interpretation.

The second important finding from this experiment is that task-related changes in brain activity were remarkably similar in patients and controls despite the difference between groups in ability to perform the tasks. The patterns of brain activity that characterized these semantic and episodic memory tasks appear not to be strongly related to successful performance of these tasks. Our results would suggest that these patterns of activity reflect the various processes involved in performing the tasks and the brain networks that mediate these processes but give little indication of how successfully these processes are able to support the ultimate behavioral outcome. This has implications for functional neuroimaging experiments in general, and those that compare groups of subjects in particular, and indicates that caution in interpreting data from such experiments is warranted. That is, similar brain activity during a particular task does not guarantee that participants are performing the task in the same way any more than equivalent performance between groups on the task guarantees that brain activity will be the same (Della-Maggiore et al., 2000). The lack of group differences in task effects also highlights the advantages of probing more deeply into imaging data, with multivariate approaches such as PLS, to reveal patterns of functional connectivity. There is now considerable evidence to suggest that these types of analyses can be informative about how similar behavioral profiles can be mediated by different underlying brain networks (Della-Maggiore et al., 2000; Stern et al., 2000; Grady et al., 2003) or how different networks can help explain multiple patterns of behavior on a given task (McIntosh et al., 1999). This type of approach can be used either in an exploratory manner or to examine specific hypotheses about functional organization, such as was done here to examine recruitment of prefrontal regions in $\mathrm{AD}$ patients.

It is also worth noting that the similarity in task-related activity in patients and controls was found despite the fact that the patients likely had a greater degree of cerebral atrophy than did the healthy controls. Thus, although atrophy can reduce measures of brain activity obtained with neuroimaging (Meltzer et al., 1990), it cannot have played a major role in our results, either in terms of task effects, which were not reduced in the patients, or in the correlational patterns that we observed, which were more extensive in the patients. Another potential confound is the effect of medications on the brain activity seen in the patients, which could have influenced their altered functional connectivity. Cholinesterase inhibitors may prevent or delay reductions in cortical metabolism as the disease progresses, including those seen in prefrontal regions (Staff et al., 2000; Nakano et al., 2001; Nobili et al., 2002), but the effect of these medications on brain activation or connectivity during cognitive tasks is unknown. However, it is unlikely that our results were attributable to the effects of cognitive enhancers in our patient sample because increased functional connectivity of prefrontal regions has been reported in unmedicated AD patients (Horwitz et al., 1995).

In conclusion, we have shown that patients with $\mathrm{AD}$ can engage additional prefrontal areas during memory task performance and that the degree of this recruitment is related to patients' ability to perform the task successfully. A similar recruitment of frontal regions has been reported during memory tasks in healthy older adults compared with younger adults (Cabeza et al., 1997; Madden et al., 1999; Reuter-Lorenz et al., 2000; Grady et al., 2002), suggesting that this might be a general re- sponse to functional loss resulting from various causes. Because all of our patients were mildly demented, recruitment of additional cortical regions is likely a response to the degenerative disease process that occurs early in its course, perhaps even before the onset of symptoms. The development of compensatory responses in relation to the evolution of early cognitive changes in $\mathrm{AD}$ should be a focus of future research in this area.

\section{References}

Backman L, Andersson JLR, Nyberg L, Winblad B, Nordberg A, Almkvist O (1999) Brain regions associated with episodic retrieval in normal aging and Alzheimer's disease. Neurology 52:1861-1870.

Becker JT, Mintun MA, Aleva K, Wiseman MB, Nichols T, DeKosky ST (1996) Compensatory reallocation of brain resources supporting verbal episodic memory in Alzheimer's disease. Neurology 46:692-700.

Binetti G, Magni E, Cappa SF, Padovani A, Bianchetti A, Trabucchi M (1995) Semantic memory in Alzheimer's disease: an analysis of category fluency. J Clin Exp Neuropsychol 17:82-89.

Braun AR, Guillemin A, Hosey L, Varga M (2001) The neural organization of discourse: an H2 15O-PET study of narrative production in English and American sign language. Brain 124:2028-2044.

Braver TS, Cohen JD, Nystrom LE, Jonides J, Smith EE, Noll DC (1997) A parametric study of prefrontal cortex involvement in human working memory. NeuroImage 5:49-62.

Buckner RL, Wheeler ME, Sheridan MA (2001) Encoding processes during retrieval tasks. J Cognit Neurosci 13:406-415.

Cabeza R, Nyberg L (2000) Imaging cognition II: an empirical review of 275 PET and fMRI studies. J Cognit Neurosci 12:1-47.

Cabeza R, Grady CL, Nyberg L, McIntosh AR, Tulving E, Kapur S, Jennings JM, Houle S, Craik FIM (1997) Age-related differences in neural activity during memory encoding and retrieval: a positron emission tomography study. J Neurosci 17:391-400.

Della-Maggiore V, Sekuler AB, Grady CL, Bennett PJ, Sekuler R, McIntosh AR (2000) Corticolimbic interactions associated with performance on a short-term memory task are modified by age. J Neurosci 20:8410-8416.

Desgranges B, Baron JC, de la Sayette V, Petit-Taboue MC, Benali K, Landeau B, Lechevalier B, Eustache F (1998) The neural substrates of memory systems impairment in Alzheimer's disease. A PET study of resting brain glucose utilization. Brain 121:611-631.

Desgranges B, Baron JC, Lalevee C, Giffard B, Viader F, de La Sayette V, Eustache F (2002) The neural substrates of episodic memory impairment in Alzheimer's disease as revealed by FDG-PET: relationship to degree of deterioration. Brain 125:1116-1124.

D’Esposito M, Detre JA, Alsop DC, Shin RK, Atlas S, Grossman M (1995) The neural basis of the central executive system of working memory. Nature 378:279-281.

Duzel E, Cabeza R, Picton TW, Yonelinas AP, Scheich H, Heinze HJ, Tulving E (1999) Task-related and item-related brain processes of memory retrieval. Proc Natl Acad Sci USA 96:1794-1799.

Edgington ES (1980) Randomization tests. New York: Marcel Dekker.

Efron B, Tibshirani R (1986) Bootstrap methods for standard errors, confidence intervals and other measures of statistical accuracy. Stat Sci 1:54-77.

Eustache F, Desgranges B, Giffard B, de la Sayette V, Baron JC (2001) Entorhinal cortex disruption causes memory deficit in early Alzheimer's disease as shown by PET. NeuroReport 12:683-685.

Frackowiak RS, Friston KJ (1994) Functional neuroanatomy of the human brain: positron emission tomography-a new neuroanatomical technique. J Anat 184:211-225.

Friston KJ, Frith CD, Liddle PF, Frackowiak RSJ (1993) Functional connectivity: the principal-component analysis of large (PET) data sets. J Cereb Blood Flow Metab 13:5-14.

Fuster JM (2000) Executive frontal functions. Exp Brain Res 133:66-70.

Giffard B, Desgranges B, Nore-Mary F, Lalevee C, de la Sayette V, Pasquier F, Eustache F (2001) The nature of semantic memory deficits in Alzheimer's disease: new insights from hyperpriming effects. Brain 124:1522-1532.

Grady CL, Haxby JV, Horwitz B, Sundaram M, Berg G, Schapiro MB, Friedland RP, Rapoport SI (1988) Longitudinal study of the early neuropsychological and cerebral metabolic changes in dementia of the Alzheimer type. J Clin Exp Neuropsychol 10:576-596. 
Grady CL, Horwitz B, Pietrini P, Mentis MJ, Ungerleider LG, Rapoport SI, Haxby JV (1996) The effect of task difficulty on cerebral blood flow during perceptual matching of faces. Hum Brain Mapp 4:227-239.

Grady CL, McIntosh AR, Beig S, Craik FIM (2001a) An examination of the effects of stimulus type, encoding strategy, and functional connectivity on the role of right prefrontal cortex in recognition memory. NeuroImage 14:556-571.

Grady CL, Furey ML, Pietrini P, Horwitz B, Rapoport SI (2001b) Altered brain functional connectivity and impaired short term memory in Alzheimer's disease. Brain 124:739-756.

Grady CL, Bernstein L, Siegenthaler A, Beig S (2002) The effects of encoding task on age-related differences in the functional neuroanatomy of face memory. Psychol Aging 17:7-23.

Grady CL, McIntosh AR, Craik FI (2003) Age-related differences in the functional connectivity of the hippocampus during memory encoding. Hippocampus, in press.

Greene JD, Hodges JR (1996) The fractionation of remote memory. Evidence from a longitudinal study of dementia of Alzheimer type. Brain 119:129-142.

Grossman M, Payer F, Onishi K, White-Devine T, Morrison D, D'Esposito M, Robinson K, Alavi A (1997) Constraints on the cerebral basis for semantic processing from neuroimaging studies of Alzheimer's disease. J Neurol Neurosurg Psychiatry 63:152-158.

Herscovitch P, Markham J, Raichle ME (1983) Brain blood flow measured with intravenous O-15 water: I. Theory and error analysis. J Nucl Med 24:782-789.

Hodges JR, Salmon DP, Butters N (1992) Semantic memory impairment in Alzheimer's disease: failure of access or degraded knowledge? Neuropsychologia 30:301-314.

Horwitz B (1994) Data analysis paradigms for metabolic-flow data: combining neural modeling and functional neuroimaging. Hum Brain Mapp 2:112-122.

Horwitz B, McIntosh AR, Haxby JV, Furey M, Salerno J, Schapiro MB, Rapoport SI, Grady CL (1995) Network analysis of PET-mapped visual pathways in Alzheimer type dementia. NeuroReport 6:2287-2292.

Jagust WJ, Friedland RP, Budinger TF, Koss E, Ober B (1988) Longitudinal studies of regional cerebral metabolism in Alzheimer's disease. Neurol 38:909-912.

Madden DJ, Turkington TG, Provenzale JM, Denny LL, Hawk TC, Gottlob LR, Coleman RE (1999) Adult age differences in the functional neuroanatomy of verbal recognition memory. Hum Brain Mapp 7:115-135.

Maril A, Wagner AD, Schacter DL (2001) On the tip of the tongue: an eventrelated fMRI study of semantic retrieval failure and cognitive conflict. Neuron 31:653-660.

McIntosh AR (1999) Mapping cognition to the brain through neural interactions. Memory 7:523-548.

McIntosh AR, Bookstein FL, Haxby JV, Grady CL (1996) Spatial pattern analysis of functional brain images using partial least squares. NeuroImage 3:143-157.

McIntosh AR, Rajah MN, Lobaugh NJ (1999) Interactions of prefrontal cortex in relation to awareness in sensory learning. Science 284:1531-1533.

Meltzer CC, Leal JP, Mayberg HS, Wagner Jr HN, Frost JJ (1990) Correction of PET data for partial volume effects in human cerebral cortex by MR imaging. J Comput Assist Tomogr 14:561-570.

Nakano S, Asada T, Matsuda H, Uno M, Takasaki M (2001) Donepezil hydrochloride preserves regional cerebral blood flow in patients with Alzheimer's disease. J Nucl Med 42:1441-1445.

Nobili F, Koulibaly M, Vitali P, Migneco O, Mariani G, Ebmeier K, Pupi A, Robert PH, Rodriguez G, Darcourt J (2002) Brain perfusion follow-up in Alzheimer's patients during treatment with acetylcholinesterase inhibitors. J Nucl Med 43:983-990.

Nyberg L, Tulving E, Habib R, Nilsson L-G, Kapur S, Houle S, Cabeza R, McIntosh AR (1995) Functional brain maps of retrieval mode and recovery of episodic information. NeuroReport 7:249-252.
Ober BA, Shenaut GK (1999) Well-organized conceptual domains in Alzheimer's disease. J Int Neuropsychol Soc 5:676-684.

Perry RJ, Hodges JR (2000) Fate of patients with questionable (very mild) Alzheimer's disease: longitudinal profiles of individual subjects' decline. Dem Geriatr Cognit Dis 11:342-349.

Reuter-Lorenz PA, Jonides J, Smith EE, Hartley A, Miller A, Marshuetz C, Koeppe RA (2000) Age differences in the frontal lateralization of verbal and spatial working memory revealed by PET. J Cognit Neurosci 12:174-187.

Sampson PD, Streissguth AP, Barr HM, Bookstein FL (1989) Neurobehavioral effects of prenatal alcohol: Part II. Partial least squares analysis. Neurotox Teratol 11:477-491.

Saykin AJ, Flashman LA, Frutiger SA, Johnson SC, Mamourian AC, Moritz CH, O'Jile JR, Riordan HJ, Santulli RB, Smith CA, Weaver JB (1999) Neuroanatomic substrates of semantic memory impairment in Alzheimer's disease: patterns of functional MRI activation. J Int Neuropsychol Soc 5:377-392.

Schreurs BG, McIntosh AR, Bahro M, Herscovitch P, Sunderland T, Molchan SE (1997) Lateralization and behavioral correlation of changes in regional cerebral blood flow with classical conditioning of the human eyeblink response. J Neurophysiol 77:2153-2163.

Smith EE, Jonides J (1998) Neuroimaging analyses of human working memory. Proc Natl Acad Sci USA 95:12061-12068.

Snodgrass JG, Vanderwart M (1980) A standardized set of 260 pictures: norms for name agreement, image agreement, familiarity and visual complexity. J Exp Psychol Hum Learn 6:174-215.

Staff RT, Gemmell HG, Shanks MF, Murray AD, Venneri A (2000) Changes in the $\mathrm{rCBF}$ images of patients with Alzheimer's disease receiving Donepezil therapy. Nucl Med Commun 21:37-41.

Stern Y, Moeller JR, Anderson KE, Luber B, Zubin NR, DiMauro AA, Park A, Campbell CE, Marder K, Bell K, Van Heertum R, Sackeim HA (2000) Different brain networks mediate task performance in normal aging and AD: defining compensation. Neurology 55:1291-1297.

Stuss DT, Benson DF (1984) Neuropsychological studies of the frontal lobes. Psychol Bull 95:3-28.

Talairach J, Tournoux P (1988) Co-planar stereotaxic atlas of the human brain. New York: Thieme.

Tulving E, Kapur S, Craik FIM, Moscovitch M, Houle S (1994) Hemispheric encoding/retrieval asymmetry in episodic memory: positron emission tomography findings. Proc Natl Acad Sci USA 91:2016-2020.

Vandenberghe R, Price C, Wise R, Josephs O, Frackowiak RSJ (1996) Functional anatomy of a common semantic system for words and pictures. Nature 383:254-256.

Wagner AD, Pare-Blagoev EJ, Clark J, Poldrack RA (2001) Recovering meaning: left prefrontal cortex guides controlled semantic retrieval. Neuron 31:329-338.

Weingartner HJ, Kawas C, Rawlings R, Shapiro M (1993) Changes in semantic memory in early stage Alzheimer's disease patients. Gerontologist 33:637-643.

Welsh K, Butters N, Hughes J, Mohs R, Heyman A (1991) Detection of abnormal memory decline in mild cases of Alzheimer's disease using CERAD neuropsychological measures. Arch Neurol 48:278-281.

Wiggs CL, Weisberg J, Martin A (1999) Neural correlates of semantic and episodic memory retrieval. Neuropsychologia 37:103-118.

Wilding EL, Rugg MD (1996) An event-related potential study of recognition memory with and without retrieval of source. Brain Cognit 119:889-906.

Woodard JL, Grafton ST, Votaw JR, Green RC, Dobraski ME, Hoffman JM (1998) Compensatory recruitment of neural resources during overt rehearsal of word lists in Alzheimer's disease. Neuropsychology 12:491-504.

Woods RP, Cherry SR, Mazziotta JC (1992) Rapid automated algorithm for aligning and reslicing PET images. J Comput Assist Tomogr 16:620-633. 